\title{
L'énigme de la supersolidité
}

Sébastien Balibar (balibar@|ps.ens.fr)

Laboratoire de Physique Statistique de l'École Normale Supérieure, 24 rue Lhomond, 75231 Paris Cedex 05, France

Un solide peut-il être superfluide (on dit alors « supersolide ») ? À très basse température, en dessous d'un dixième de kelvin, I'hélium solide est élastique comme tout solide, mais une partie de sa masse semble couler sans dissipation à travers le reste.

Malgré l'intense activité de nombreux groupes de recherche et la certitude acquise que cette supersolidité vient du désordre des échantillons étudiés, on est loin de comprendre son origine précise.

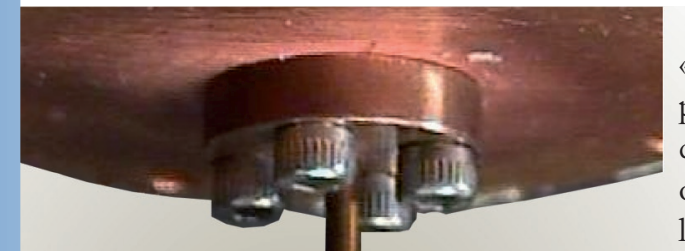

"Supersolide " signifie " superfluide " et " solide " à la fois et, au début, cela me paraissait un peu carpe et lapin, une chimère. Lorsqu'en 2004 Kim et Chan ont publié deux articles [1] proposant que l'hélium solide pouvait être supersolide, je n'y ai donc pas cru. Je pouvais difficilement admettre que les atomes d'hélium puissent être à la fois localisés aux noeuds d'un réseau cristallin et délocalisés comme dans l'onde macroscopique que constitue un superfluide.

Pourtant, les dernières expériences présentées par le groupe de Chan pendant l'été 2008 montrent qu'il y a dans l'hélium solide en dessous de 0,1 kelvin, assez de masse localisée pour conférer au système l'élasticité d'un solide mais, simultanément, une fraction de la masse qui coule sans dissipation. En 2006, nous avons montré que le désordre jouait un rôle essentiel dans cette affaire, en particulier les joints entre grains cristallins dans des échantillons polycristallins [2]. Mais cela ne permettait pas de comprendre l'ensemble des observations effectuées dans toutes sortes d'échantillons préparés différemment, ni surtout de comprendre comment le désordre permet la superfluidité observée. Il y a vraiment un problème aussi mystérieux que fondamental là-dessous.

\section{L'observation initiale de Kim et Chan}

Qu'ont donc observé Kim et Chan de si extraordinaire à l'Université de Pennsylvanie ? Ils ont suspendu une petite boîte à un axe en bronze pour en faire un pendule de torsion (fig. 1). C'est une méthode classique pour détecter la superfluidité. En effet, quand on remplit une telle boîte d'hélium liquide et que celui-ci devient superfluide (à 2,2 K), la viscosité disparait et les parois de la boîte oscillante cessent d'entraîner le liquide intérieur. Comme le moment d'inertie diminue, la période propre du pendule aussi. En mesurant cette période, on mesure donc la fraction superfluide de l'échantillon. Mais Kim et Chan ont observé un effet semblable avec de l'hélium solide. Vers $0,1 \mathrm{~K}$, la période passait à une valeur inférieure, 

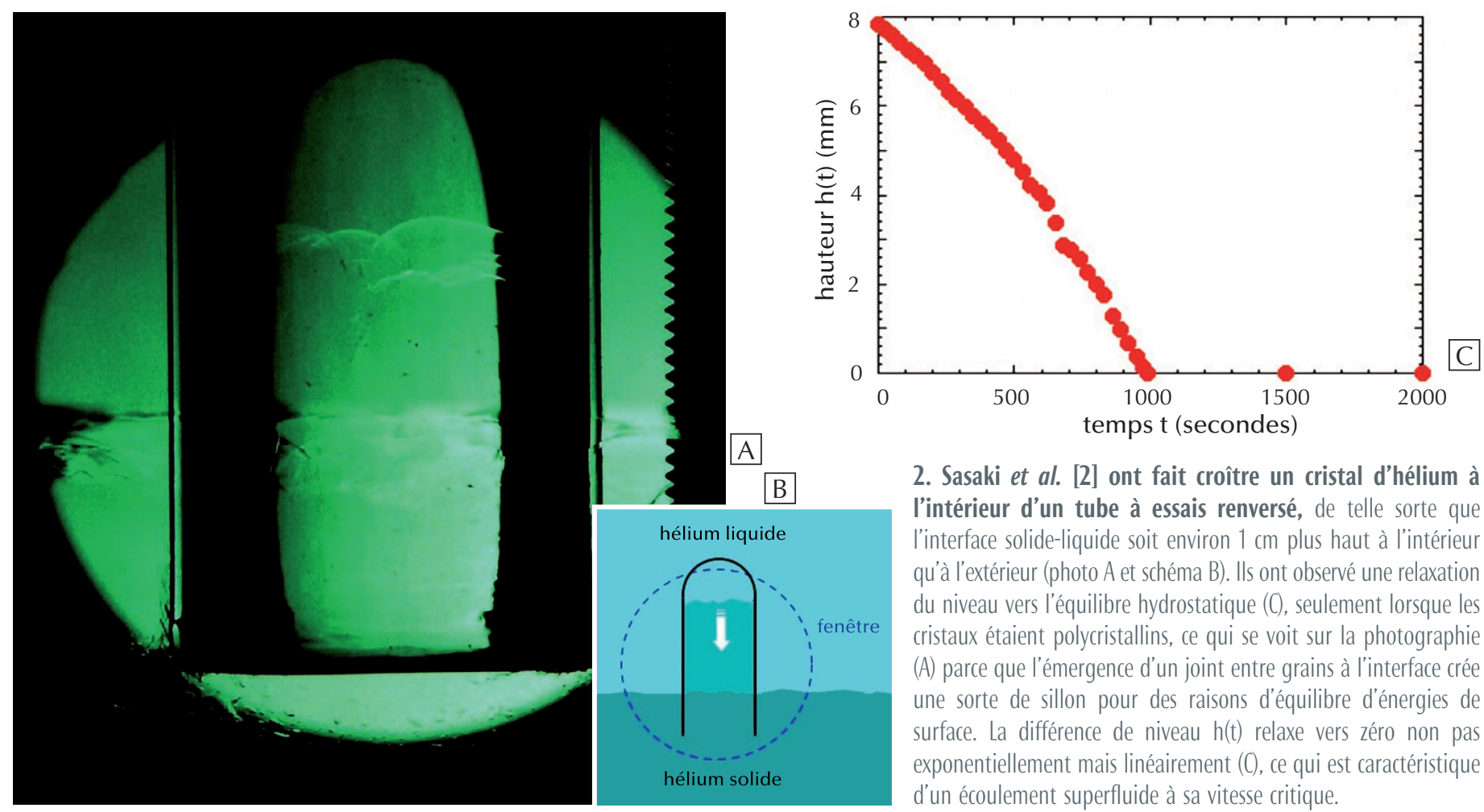

2. Sasaki et al. [2] ont fait croître un cristal d'hélium à l'intérieur d'un tube à essais renversé, de telle sorte que l'interface solide-liquide soit environ $1 \mathrm{~cm}$ plus haut à l'intérieur qu'à l'extérieur (photo A et schéma B). Ils ont observé une relaxation du niveau vers l'équilibre hydrostatique (C), seulement lorsque les cristaux étaient polycristallins, ce qui se voit sur la photographie (A) parce que l'émergence d'un joint entre grains à l'interface crée une sorte de sillon pour des raisons d'équilibre d'énergies de surface. La différence de niveau $h(t)$ relaxe vers zéro non pas exponentiellement mais linéairement (C), ce qui est caractéristique d'un écoulement superfluide à sa vitesse critique.

comme si $1 \%$ de la masse d'hélium devenait superfluide. Ils ont évidemment vérifié qu'avec une boîte vide il ne se passait rien. Rien non plus s'ils remplaçaient l'hélium 4 (des bosons) par de l'hélium 3 (des fermions). Enfin l'effet disparaissait audelà d'une vitesse critique, comme avec un superfluide ordinaire. Face à cet ensemble de résultats convergents, ils ont proposé que l'hélium était supersolide en dessous de $0,1 \mathrm{~K}$.

\section{Le point de vue des théoriciens}

La supersolidité était-elle donc possible ? À ce stade, il faut dire que la controverse couvait entre théoriciens depuis 35 ans. Elle remonte même à 1956, lorsque Penrose et Onsager ont généralisé la condensation de Bose-Einstein à la matière condensée (à l'origine, elle avait été introduite pour les gaz parfaits). Penrose et Onsager ont identifié sa signature sous forme de ce qu'on appelle un " ordre non diagonal à longue distance dans la matrice densité ". Cela signifie que la fonction d'onde du système est cohérente à l'échelle macroscopique, et c'est l'origine de la superfluidité. Mais ils ont expliqué que cet ordre était impossible dans un solide où la matière est localisée et ne s'échange donc pas à grande distance. Toutefois, en 1970, Chester a remarqué que les fonctions d'ondes utilisées par Penrose et Onsager n'étaient pas symétrisées, ce qui est incorrect pour des bosons.

Un solide pouvait-il donc être superfluide ? C'est ce qu'ont proposé Thouless [3], puis Andreev et Lifshitz [4], en 1969. Dans un cristal classique, déplaçons un atome pour ménager un trou (une "lacune») sur l'un des sites du réseau. Cela coûte une certaine énergie $E_{0}$, car on coupe des liaisons attractives entre atomes. Mais supposons que, dans ce cristal, les fluctuations quantiques des atomes soient grandes. Dans un tel cristal quantique, les atomes sont mal localisés et peuvent s'échanger par effet tunnel. Pour une lacune, l'échange avec un atome voisin devrait être encore plus facile et s'effectuer avec une fréquence assez grande. Une lacune quantique devient donc une onde délocalisée, comme les électrons dans un métal, et son énergie est à l'intérieur d'une bande autour de $\mathrm{E}_{0}$. Plus la fréquence d'échange est grande, plus le bas de cette bande descend. Si ce bas de bande est négatif (le cristal sans lacune étant pris comme zéro d'énergie), l'état fondamental du cristal est surprenant : les lacunes envahissent le cristal jusqu'à ce que, si les interactions entre lacunes sont répulsives, on cesse de gagner de l'énergie en en mettant davantage. Le cristal est alors "incommensurable ", car le nombre d'atomes n'est pas égal au nombre de sites. Enfin, si les atomes sont des bosons, les lacunes aussi, et le fluide de lacunes doit devenir superfluide en dessous d'une température critique qui dépend de leur densité. Un flux de lacunes étant équivalent à un flux d'atomes en sens inverse, ce cristal incommensurable devrait admettre un écoulement superfluide de matière en son sein, avoir des tourbillons quantifiés donc des propriétés de rotation anormales, être, en somme, supersolide. 
3. On obtient des cristaux de qualité extrêmement variable selon la méthode de croissance. Par exemple, d'excellents monocristaux à partir du superfluide à très basse température (A) ou une véritable neige de flocons d'hélium enchevêtrés par croissance rapide à partir du liquide normal à relativement haute température (B). II est probable que le désordre responsable de la supersolidité n'est pas le même pour des échantillons aussi différents : dislocations peutêtre dans le premier cas (A), joints de grains et inclusions liquides ou vitreuses dans le second (B).

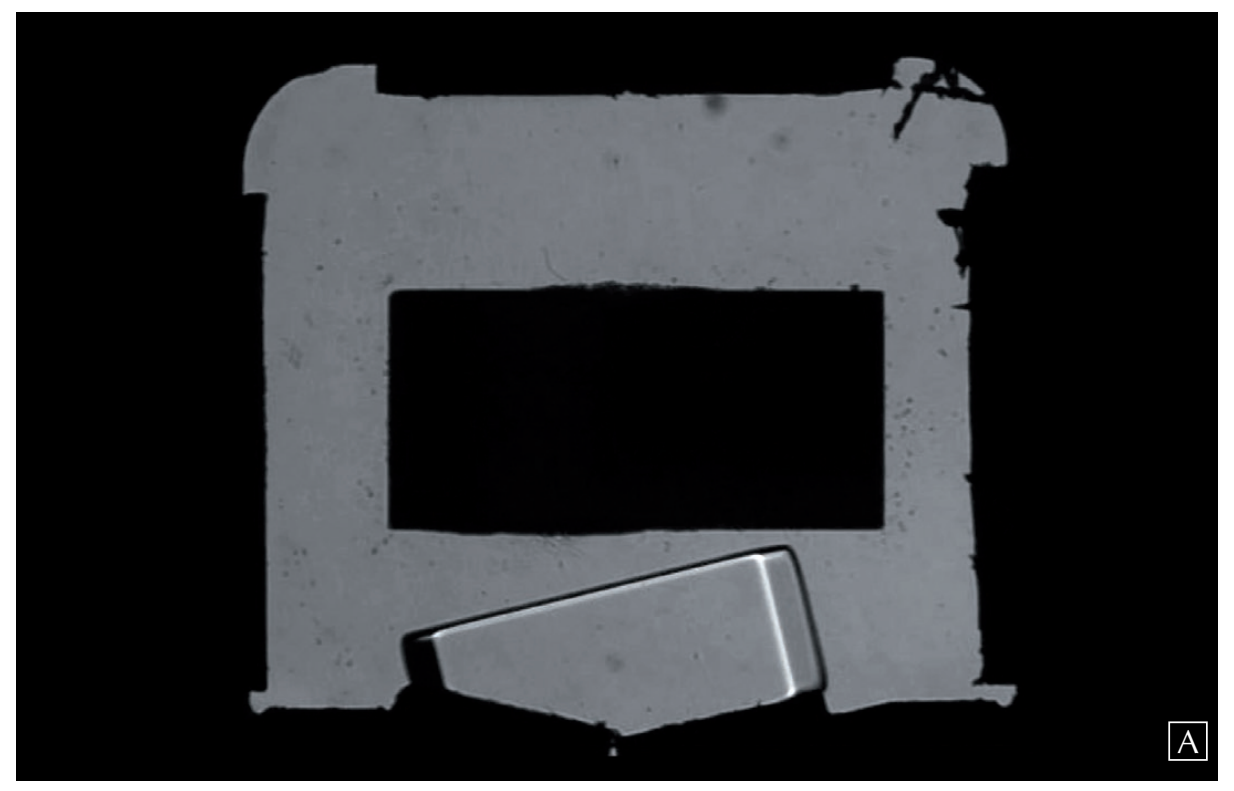

\〉

Ce que Kim et Chan avaient observé était-il donc ce cristal quantique imaginé par Thouless, Andreev et Lifshitz ? Il s'avéra assez rapidement que non. $1 \%$ de fraction supersolide aurait nécessité, en gros, 1\% de lacunes et, malgré quelques objections d'Anderson, Brinkman et Huse [5], cela semble incompatible avec les expériences comme avec les simulations numériques, qui trouvent toutes que le bas de la bande des lacunes est vers $+10 \mathrm{~K}(\sim 1 \mathrm{meV})$. Dans un cristal parfait, la densité de lacunes est donc négligeable en dessous de $1 \mathrm{~K}$.

La publication des expériences de Kim et Chan a déclenché de nouvelles polémiques, mais je crois exclu qu'un cristal d'hélium parfait puisse être supersolide à la ThoulessAndreev-Lifshitz. Il est apparu que le désordre des échantillons étudiés devait jouer un rôle important.

\section{Le rôle du désordre}

Deux nouvelles expériences ont en effet été effectuées. À l'Université de Cornell (USA), Rittner et Reppy ont refait l'expérience de Kim et Chan et observé des anomalies semblables [6]. Puis, ils ont réchauffé leur échantillon près de la température de fusion avant de le refroidir lentement, effectuant ainsi un " recuit " afin d'éliminer dislocations, joints de grains et autres défauts. Après recuit, les anomalies de rotation avaient disparu. Au contraire, tremper leur hélium produisait des échantillons très désordonnés où la fraction supersolide pouvait atteindre $20 \%$ ! On comprit donc pourquoi la fraction supersolide pouvait varier de 0,03 à $20 \%$ d'une expérience à l'autre : cela dépendait du désordre produit par chaque méthode de solidification.
À l'ENS (Paris), nous avons cherché une preuve directe de la supersolidité : si la matière coulait vraiment à travers le solide, on devait pouvoir faire une expérience de vases communicants. Nous avons donc mis un tube à essais renversé dans une cellule remplie d'hélium solide en équilibre avec son liquide (fig. 2A et B). Nous avons fait en sorte que le niveau de l'interface solide-liquide soit plus haut d'un centimètre à l'intérieur du tube qu'à l'extérieur et nous avons regardé ce qui se passait. C'est simple, à condition de disposer d'un cryostat optique avec de grandes fenêtres traitées pour laisser passer la lumière visible sans réchauffer la cellule expérimentale, même jusque vers 40 millikelvins. Solide ou liquide, l'hélium conduit remarquablement la chaleur, la température est donc très homogène et la forme des cristaux contrôlée par la gravité : plus dense, le solide est au fond. Si les niveaux ne sont pas les mêmes des deux côtés, ils devraient tendre à relaxer l'un vers l'autre. Pour cela, il faut que le cristal fonde à l'intérieur du tube et croisse à l'extérieur. Mais comme le solide est plus dense que le liquide et que le tube est fermé en haut, il faut transférer de la matière de l'intérieur vers l'extérieur du tube, à travers le solide. Donc, si l'hélium n'est pas supersolide les niveaux ne peuvent pas relaxer. Mais s'il l'est, un équilibre hydrostatique s'établit dans le solide comme s'il s'agissait d'un liquide et les niveaux relaxent (fig. 2C). Or nous avons montré qu'il y a transfert de masse seulement si le solide est polycristallin, c'est-à-dire s'il est fait de plusieurs grains [2]. Nous avons donc éliminé la possibilité d'un effet intrinsèque à la Thouless-Andreev-Lifshitz, qui serait dû à l'incommensurabilité des cristaux. 


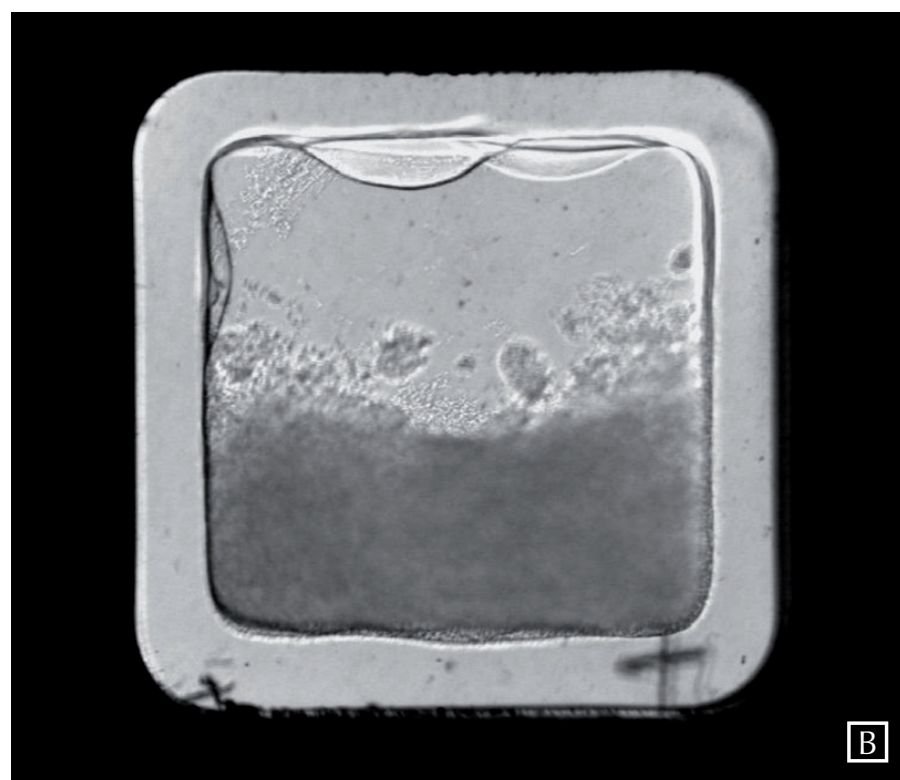

Ensuite, il était tentant d'imaginer que la matière coulait le long des joints de grains, d'autant que Pollet et al. [7] ont simulé la physique de ces joints et prédit que ce sont des systèmes bidimensionnels d'épaisseur microscopique (environ $1 \mathrm{~nm}$ ), qui sont désordonnés et supersolides en dessous d'une fraction de kelvin. Je dis bien "supersolide " et pas "superfluide " parce que ces joints, d'après Pollet et al., ne sont pas liquides : d'une part, la matière susceptible de s'y écouler n'est qu'une faible fraction de la matière totale, d'autre part, les grains ne peuvent pas glisser les uns par rapport aux autres.

Entamant alors une étude systématique de ces joints de grains, nous avons montré que leur épaisseur est bien microscopique [2] et qu'ils ne sont pas liquides (c'est une question de " prémouillage " qui est importante en science des matériaux et mériterait un autre article). Mais nous ne savons pas encore s'ils sont vraiment supersolides. En effet, nous avons aussi montré que les échantillons polycristallins contiennent des canaux qui, eux, sont liquides, là où les joints de grains rencontrent les parois de la cellule ou se rencontrent entre eux. Il est donc possible que, dans notre expérience, le transport de matière se soit effectué le long de ces canaux liquides et pas le long des joints de grains euxmêmes. Nous préparons d'autres expériences pour trancher cette question.

Malgré tout, était-il possible que, dans l'expérience de Kim et Chan, la matière découplée des parois soit simplement l'intérieur de ces joints ? Il aurait fallu qu'ils contiennent au moins 1\% de la masse totale. A priori, leurs échantillons devaient être des polycristaux. Pour obtenir $1 \%$ avec des joints de $1 \mathrm{~nm}$ d'épaisseur dont au plus un tiers est superfluide, il aurait fallu des grains d'environ $100 \mathrm{~nm}$. Nous avons donc étudié le désordre des échantillons en fonction de leurs conditions de croissance [2] et trouvé qu'il était extrêmement variable (fig. 3). Cristallisés à pression constante à partir du superfluide, les cristaux sont presque parfaits. Mais, cristallisé à volume constant et à relativement haute température, l'hélium est polycristallin avec des grains de taille micronique. Une trempe à la manière de Rittner et Reppy produit de la neige d'hélium. Comme ces méthodes conduisent à une supersolidité variable, je crois que, selon les cas, les défauts responsables sont des dislocations, c'est-à-dire des défauts du réseau à l'intérieur de monocristaux, des joints de grains dans des échantillons polycristallins, ou même des parties liquides ou vitreuses dans des échantillons trempés.

À ce stade, on a réexaminé différents artefacts possibles : Dash suggéra par exemple, que les cristaux glissaient le long des parois ; mais une boîte cubique donnait les mêmes résultats qu'une boîte cylindrique. Quant à de Gennes, il invoqua un changement de mobilité des défauts. Je crois aujourd'hui qu'il était sur la bonne piste, mais que l'effet est l'opposé de ce qu'il croyait. Dans un cristal classique, un défaut peut se déplacer sous l'effet d'une contrainte, mais le mouvement est dissipatif et irréversible car il procède par activation thermique. Dans un cristal quantique, le mouvement des défauts correspond à des sauts par effet tunnel de site en site, qui sont réversibles. L'idée de de Gennes était que la mobilité des défauts devenait quantique, donc grande à basse température, ce qui conférait au solide une superplasticité qui modifiait les propriétés de rotation. Mais, en 2007, Day et Beamish mesurèrent le module de cisaillement de l'hélium solide et leurs résultats furent pour le moins surprenants [8].

\section{Le mystère de la rigidité de l'hélium supersolide}

On se serait attendu à ce que, si une partie de la masse pouvait couler, le solide ressemble à un liquide et que sa rigidité globale diminue. Or, Day et Beamish ont trouvé le contraire : à la température où sa rotation devient anormale, l'hélium solide devient plus rigide, son module de cisaillement augmente de 8\% (fig. 4) ! Pour mesurer ce module, on applique une contrainte de cisaillement et on mesure la déformation produite. Le module est le rapport des deux. Sans défauts, ce module est une propriété intrinsèque du réseau cristallin. Mais les défauts, en se déplaçant, peuvent produire une déformation additionnelle et, si leur déplacement (quantique) est réversible, le cristal présente une rigidité effective plus faible. Supposons alors qu'il s'agisse de dislocations et qu'aux environs de $0,1 \mathrm{~K}$ le piégeage d'impuretés (de l'hélium 3 résiduel) bloque leur mouvement : la rigidité effective du solide augmente. Dans le cadre de ce modèle, Day et Beamish ont trouvé une énergie de liaison des atomes d'hélium 3 aux dislocations en accord avec des mesures anciennes $(0,7 \mathrm{~K}$, soit $0,06 \mathrm{meV})$. Il semble donc bien que la rigidité varie à cause du piégeage des dislocations par les impuretés. Mais comment se fait-il que ce piégeage s'accompagne d'une apparente supersolidité ? 
4. Day et Beamish [6] ont découvert que le module de cisaillement de l'hélium solide augmente à basse température d'environ $8 \%$ (points rouges). Cette variation en température, une fois normalisée, est exactement la même que la variation de l'inertie de rotation de l'hélium solide (NCRI, cercles noirs) observée par Kim et Chan [1]. II serait donc surprenant que les deux phénomènes n'aient pas une origine commune. Mais pourquoi ? C'est tout le problème qui se pose aujourd'hui.

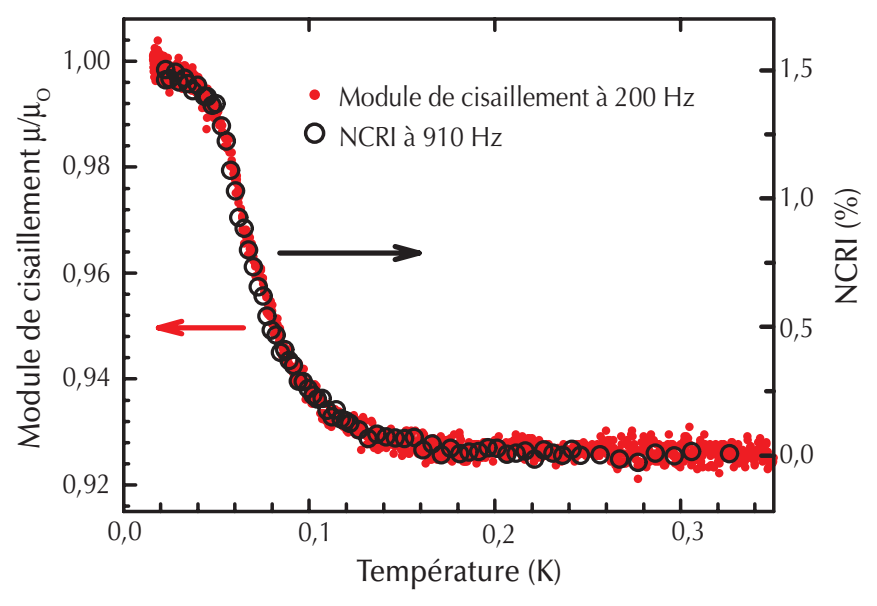

$\gg$

On pensa alors à un artefact assez simple. La période de l'oscillateur de torsion dépend certes de la masse qui oscille, mais aussi de la force de rappel élastique du dispositif. Or cette force dépend essentiellement de l'élasticité de l'axe en bronze, mais un peu aussi de celle de l'hélium. L'augmentation de la rigidité de l'hélium pouvait-elle expliquer la faible variation de période observée ? Chan et ses élèves ont vérifié que non en construisant, fin 2007, un oscillateur très rigide où la contribution de l'hélium à l'élasticité de l'ensemble était vraiment négligeable. Ils ont encore trouvé un changement de période mesurable. C'était donc bien l'inertie qui changeait. De plus, Rittner et Reppy ont testé avec précision l'écoulement intérieur à la boîte. Dans leur boîte, l'hélium était confiné entre deux cylindres concentriques. L'espace mince entre les cylindres pouvait être bloqué en insérant un obstacle le long d'une génératrice. Avec un espace libre ils observèrent un changement de période. Avec un espace bloqué, l'effet disparaissait. Les anomalies de rotation étaient donc bien dues à un écoulement d'ensemble. Il ne pouvait pas s'agir de petits réarrangements locaux de la matière!

Il fallut donc affronter l'incompréhensible : comment le piégeage des défauts pouvait-il conditionner la supersolidité de l'ensemble? Parmi les scénarios proposés récemment, il en est un qui n'est pas encore vérifié mais qui me semble simple et intéressant. Restreignons-nous au monocristal contenant des dislocations. Des calculs de Pollet ont montré que le cœur des dislocations pouvait être supersolide. À condition que les dislocations soient connectées entre elles, tout le cristal pourrait devenir supersolide si la cohérence quantique percole le long des cœurs à travers le réseau de dislocations. À suffisamment basse température cela semble possible. Il resterait difficile d'atteindre une fraction superfluide de $0,1 \%$ avec une densité de dislocations raisonnable, mais Biroli et Bouchaud pensent que les fluctuations transverses des dislocations aident à cela. Mais pourquoi cette percolation de la cohérence quantique s'accompagne-t-elle d'une augmentation de rigidité ?

Svistunov a eu l'idée intéressante suivante. Si les atomes d'hélium 3 sont liés aux dislocations, ils devraient l'être davantage au croisement de deux dislocations. On pourrait donc imaginer que les hélium 3 forcent les dislocations à se croiser, c'est-àdire à se connecter. La température de transition superfluide serait en fait la température à laquelle le réseau de dislocations se connecte! Il faudrait comparer les énergies en jeu avec l'interaction élastique entre dislocations, mais l'idée a le mérite d'être simple. Voilà en tout cas où nous en sommes, face à une énigme dont on cherche toujours la clef.

Et maintenant ? La mobilité des défauts devrait être différente selon qu'elle s'opère parallèlement ou perpendiculairement aux plans hexagonaux de la structure cristalline ; la supersolidité devrait donc être sensible à l'orientation des cristaux. En collaboration avec l'équipe de Chan, nous construisons un oscillateur de torsion transparent où nous pourrons voir cette orientation. Dans une autre cellule transparente, nous étudierons comment la rigidité transverse de l'hélium solide dépend de la présence de joints de grains, de dislocations, et de l'orientation des cristaux lorsqu'ils sont monocristallins. Nous espérons donc résoudre cette énigme en 2009 ... sauf que, depuis 4 ans que ce problème est posé, nous avons appris à rester prudents dans nos espérances [9]. 